\title{
Relevance between GerdQ score and the severity of reflux esophagitis in Uygur and Han Chinese
}

\author{
Man Wang ${ }^{1, *}$, Jing-Zhan Zhang ${ }^{2, *}$, Xiao-Jing Kang $^{2}$, Li Li $^{1}$, Xiao-Ling Huang ${ }^{1}$, \\ Kuerbanjiang Aihemaijiang ${ }^{1}$, Aheman Ayinuer ${ }^{1}$, Yue-Xian Li $^{1}$, Xiao-Lei He $^{1}$ and \\ Feng Gao ${ }^{1}$ \\ ${ }^{1}$ Department of Gastroenterology, People's Hospital of Xinjiang Uygur Autonomous Region, Urumqi, China \\ ${ }^{2}$ Department of Dermatology, People's Hospital of Xinjiang Uygur Autonomous Region, Urumqi, China \\ *These authors have contributed equally to this work \\ Correspondence to: Feng Gao, email: drxjgf@sina.com \\ Keywords: reflux esophagitis, gastroesophageal reflux disease questionnaire, endoscopy \\ Received: December 27, $2016 \quad$ Accepted: May 29, $2017 \quad$ Published: August 10, 2017 \\ Copyright: Wang et al. This is an open-access article distributed under the terms of the Creative Commons Attribution License 3.0 \\ (CC BY 3.0), which permits unrestricted use, distribution, and reproduction in any medium, provided the original author and source \\ are credited.
}

\section{ABSTRACT}

Gastroesophageal reflux disease questionnaire (GerdQ) was used to investigate the inpatients with typical reflux related symptoms in Gastroenterology. According to heartburn, regurgitation, abdominal pain, nausea, sleep disorders, whether taking over the counter (OTC) drugs 6 points to score. Using endoscopy as the gold standard for the diagnosis of reflux esophagitis (RE), and the results were compared with GerdQ score to determine the threshold value for $R E$, to analyze the distribution of GerdQ score for patients with RE, to assess the relationship between the GerdQ score and the severity of RE. A total of 1233 patients were enrolled in this study, including 538 patients had RE and 695 had not. There was statistical significance in the GerdQ score of RE group and non-RE group $(P<0.05)$, showing that significant correlation between the score and the occurrence of RE. GerdQ score and the severity of RE were positively correlated. Further research also showed that there was a direct correlation between GerdQ score and the severity of RE in the Uygur and Han. GerdQ seems to be an useful screening tool in initial diagnosis of RE, and positively correlated with the severity of RE.

\section{INTRODUCTION}

Reflux esophagitis (RE) is defined as reflux of the gastric and (or) duodenum contents into the esophagus causes a range of mucosal breaks, erosions or ulcers within the esophagus. RE is a prevalent, chronic and relapsing upper digestive disease, especially in recent years. With the change of people's diet structure and lifestyle, the morbidity of RE is increasing greatly in the world [1-3], which has significantly compromised the quality of life for patients. Gastroesophageal reflux disease questionnaire (GerdQ) is designed by Dent et al [4] in 2007, it is a selfadministered diagnostic questionnaire consisted by six items. It's mainly used as a tool to improve and standardise symptom-based diagnosis and evaluation the treatment effects in patients with GERD [5]. The questionnaire is simple, convenient, non-invasive examination, low price, good patient compliance, can be completed in the clinic [6]. The diagnostic validity and reliability of GerdQ has been confirmed [7-10]. In order to understand the occurrence rates of reflux related symptoms, to explore the correlation between the endoscopic manifestations and GerdQ score, and to provide reference for clinical diagnosis of RE, we conducted the study.

\section{RESULTS}

General characteristics of the patients

A total of 1398 patients were collected for the study, of whom 1233 were enrolled in the final analysis. A flowchart of the study, subject withdrawal at various 
stages and the final diagnoses are shown in Figure 1. Among the 1233 patients, 532 were males and 701 were females. Their mean age was $53.72 \pm 11.99$ years (range 1881 years). Their mean body mass index (BMI) was $25.34 \pm$ $3.73 \mathrm{~kg} / \mathrm{m}^{2}$. There were 482 were Uygur and 751 were Han patients, among whom 538 were RE and 695 were nonRE. In Uygur, 234 were RE patients and 248 were nonRE. In the Han, 304 cases were RE, and 447 were nonRE. The basic characteristics of these patients in RE group and non- RE group were shown in Table 1. The incidence of male patients in the RE group was significantly higher than male patients in the non-RE group $\left(\chi^{2}=23.567\right.$, $P<0.01)$. There were no difference between the RE and the non-RE groups in age $(t=-0.884, P>0.05)$, BMI $(t=1.190$, $P>0.05)$ and educational level $\left(\chi^{2}=0.307, P>0.05\right)$, but $83.09 \%$ RE patients had education level beyond secondary school. The Uygur and Han in the RE group and non-RE group account for the proportion had significant difference $\left(\chi^{2}=7.771, P<0.01\right)$, the detection rate of RE in the Uygur was higher than that in the Han.

\section{The GerdQ cut-offs}

It is noted in patients with a GerdQ sum score between 0 and 14 , the RE show direct correlation with the increasing of GerdQ cut-off scores. In those with a sum score of 8-10,
$61.01 \%$ had RE, and in those with a sum score of 3-7, $15.94 \%$ had RE. However, none with a score of $0-2$ had RE.

\section{RE classification and GerdQ scores}

Of the 538 patients with RE, nearly 412 cases (76.58\%) were demonstrated as grade A lesion, 76 cases $(14.12 \%)$ were categorized as grade B, 32 cases $(5.95 \%)$ were grade $\mathrm{C}$, and only 18 cases $(3.35 \%)$ were grade $\mathrm{D}$ lesion. The distribution of GerdQ scores in all patients is shown in Table 2. The mean GerdQ score of patients with RE and non-RE were 10.08 \pm 2.89 and 9.11 \pm 3.52 , respectively, and had significant difference between the two groups $(t=2.33, P<0.05)$. All of the RE patients were graded according to LA classification, showed that the mean GerdQ scores of patients with LA-A, LA-B, LA-C and LA-D was $10.48 \pm 2.91,11.75 \pm 2.77,11.80 \pm 2.98$ and $13.35 \pm 2.95$, respectively. Thus it can be seen, the total GerdQ score increased with increasing severity of esophageal mucosal defect. Spearman correlation analysis showed that the GerdQ score was positively correlated with the severity of RE $(r=0.244, P<0.01)$. The mean GerdQ score of patients with Uygur and Han was $10.23 \pm 3.17$ and $10.03 \pm 2.72$, respectively, Spearman rank correlation analysis showed that there was a direct correlation between the GerdQ score and the severity of

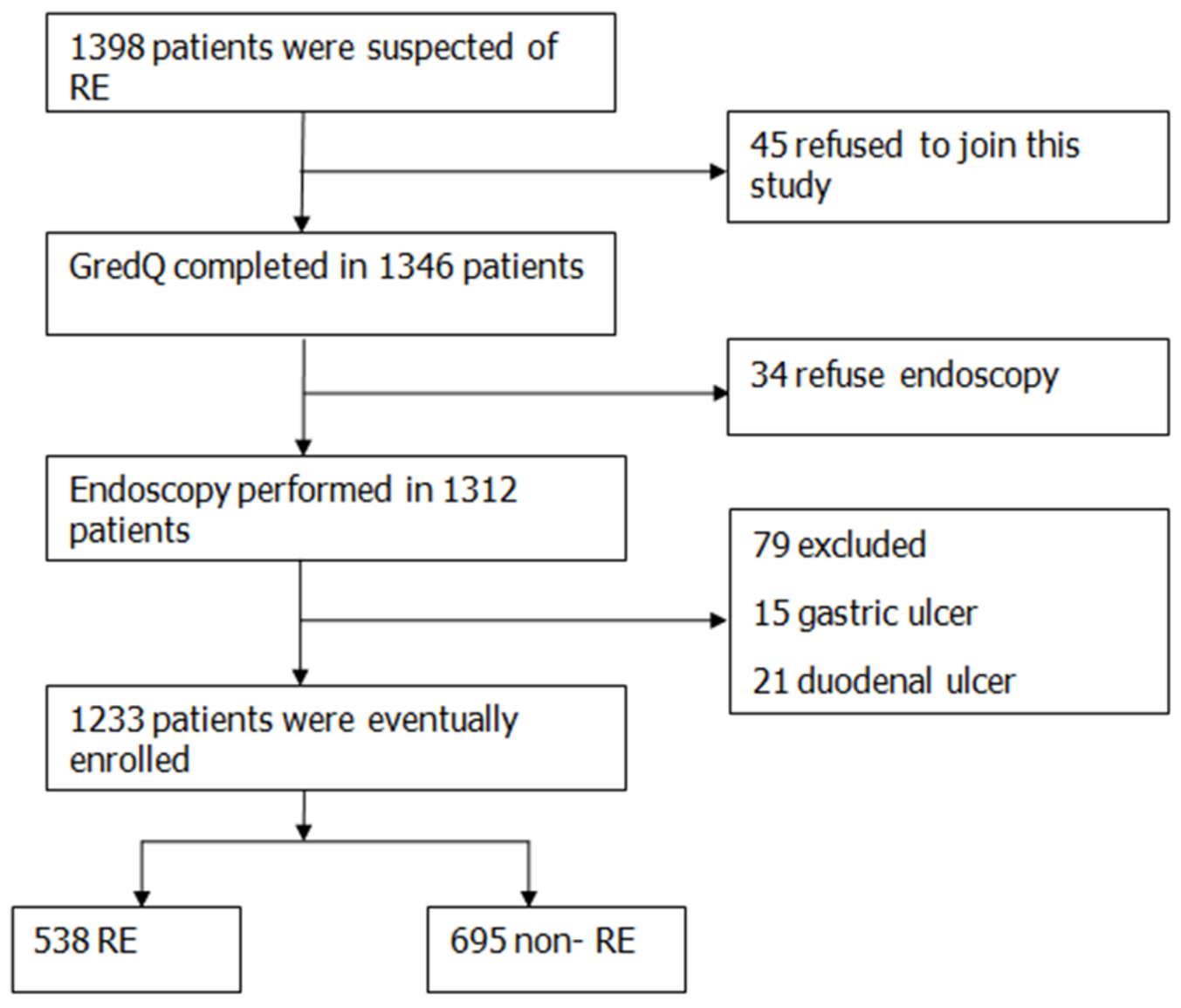

Figure 1: Flow chart of the patients' enrollment. 
Table 1: Characteristic of RE group and non-RE group

\begin{tabular}{lcccc}
\hline Items & RE & Non-RE & Test statistic & $P$ value \\
\hline Total number (n) & 538 & 695 & & 0 \\
Gender, Male [n (\%)] & $274(50.90)$ & $258(37.08)$ & $\chi^{2}=23.567$ & $t=-0.884$ \\
Age (mean \pm SD, yr) & $53.46 \pm 12.28$ & $55.13 \pm 10.34$ & $t=1.190$ & 0.378 \\
BMI (mean \pm SD) & $25.45 \pm 3.74$ & $24.72 \pm 3.66$ & $\chi^{2}=7.771$ & 0.235 \\
Ethnic [n(\%)] & & & & 0.005 \\
Uygur & $234(48.55)$ & $248(51.45)$ & & \\
Han & $304(40.48)$ & $447(59.52)$ & & \\
Education level [n(\%)] & & & & \\
Primary school & $91(16.91)$ & $120(17.26)$ & & \\
Secondary school & $267(49.63)$ & $334(48.06)$ & & \\
College & $180(33.46)$ & $241(34.68)$ & & \\
\hline
\end{tabular}

Table 2: Relationship of RE grade and symptomatic scores

\begin{tabular}{lccc}
\hline Grading of RE & Cases $(\mathbf{n})$ & Range & GerdQ score \\
\hline The non-RE & 695 & $2 \sim 18$ & $9.11 \pm 3.52$ \\
The RE & 538 & $3 \sim 18$ & $10.08 \pm 2.89$ \\
LA grade A & 412 & $3 \sim 18$ & $10.48 \pm 2.91$ \\
LA grade B & 76 & $6 \sim 18$ & $11.75 \pm 2.77$ \\
LA grade C & 32 & $6 \sim 18$ & $11.80 \pm 2.98$ \\
LA grade D & 18 & $7 \sim 17$ & $13.35 \pm 2.95$ \\
\hline
\end{tabular}

$\mathrm{RE}$ in the Uygur and Han $(r=0.233, P<0.05 ; r=0.201$, $P<0.05)$.

\section{Diagnostic value of the GerdQ}

With the increasing of critical value of GerdQ, the sensitivity decreased gradually, and the specificity increased. Suggesting that Gerd Q is a good diagnostic tool. When the cut-off score was 9, the Youden index reached a maximum of 0.53 , in which the area under the ROC was 0.736 , the ROC analysis gave the optimal balance between sensitivity $(87.7 \%)$ and specificity $(65.7 \%)$ for RE, it showed that the questionnaire had high credibility (Figure 2).

\section{DISCUSSION}

$\mathrm{RE}$ is a chronic, recurrent disease commonly recognized in developed countries and developing countries. The disease manifests itself with varying severity, from mild to incapacitating, with significant impairment of quality of life [12]. The cardinal symptoms are considered to be heartburn and regurgitation. Furthermore, the burden of the disease is large and affects the quality of life for patients, resulting in both direct health care related costs as well as indirect costs due to loss [13]. RE is a symptom based disease, and more and more people pay attention to the symptom based questionnaire. GerdQ scale provides us a simple, reliable and effective diagnostic method, and it has high clinical application value.

Among the 538 patients with RE, including 274 males, the proportion of males was higher than that of males with non-RE (including 258 males), showing that male patients are more likely to develop into RE, which is consistent with Vakil $\mathrm{N}$ et al [14] and Hu et al [15]. This suggested that we should strengthen management of male patients to reduce the incidence of RE. Compared with the non-RE group, $83.09 \%$ patients with the level of education were Secondary school or above in the RE group. The result was consistent with the research of Bai et al [7], which indicates that people with higher educational level are more concerned about their own health. Suggesting that we should strengthen the popularization of RE related medical knowledge, seminars, Conduct lectures, free 
Table 3: The GerdQ questionnaire

\begin{tabular}{|c|c|c|c|c|}
\hline \multirow{2}{*}{ Questions } & \multicolumn{4}{|c|}{ Frequency score (points) for symptoms } \\
\hline & 0 day & 1 day & 2 3days & 4 7days \\
\hline $\begin{array}{l}\text { How often did you have a burning feeling behind your } \\
\text { breastbone (heartburn)? }\end{array}$ & 0 & 1 & 2 & 3 \\
\hline $\begin{array}{l}\text { How often did you have stomach contents (liquid or food) } \\
\text { moving upwards to your throat or mouth (regurgitation)? }\end{array}$ & 0 & 1 & 2 & 3 \\
\hline $\begin{array}{l}\text { How often did you have pain in the center of the upper } \\
\text { stomach? }\end{array}$ & 3 & 2 & 1 & 0 \\
\hline How often did you have nausea? & 3 & 2 & 1 & 0 \\
\hline $\begin{array}{l}\text { How often did you have difficulty getting a good night's } \\
\text { sleep because of your heartburn and/or regurgitation? }\end{array}$ & 0 & 1 & 2 & 3 \\
\hline $\begin{array}{l}\text { How often did you take additional medication for your } \\
\text { heartburn and/or regurgitation, other than what the } \\
\text { physician told you to take (such as Tums, Rolaids and } \\
\text { Maalox)? }\end{array}$ & 0 & 1 & 2 & 3 \\
\hline
\end{tabular}

medical consultation clinic and other propagandas, to improve the patient's awareness of RE.

On the other hand, the incidence of RE in Uygur patients was significantly higher than that in Han, suggesting that the characteristics of RE in different ethnic groups were different. Munila et al [16] conducted a survey of the prevalence of GERD in the Han and Uygur physical examination population and found that the incidence rate of GERD in Uygur was higher than that in Han. This difference may leaded by different national

\section{ROC Curve}

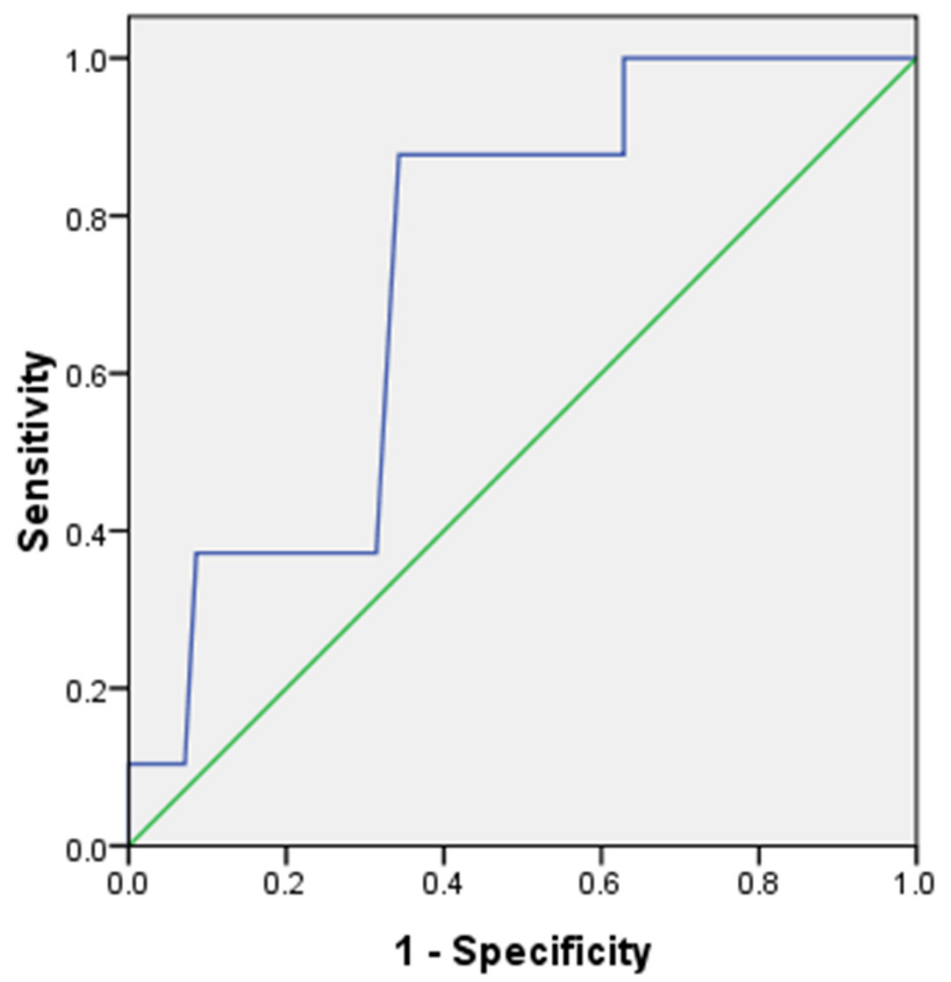

Figure 2: ROC curve of the GerdQ. Specificity and sensitivity by cut-off score. 
culture, religious belief, lifestyle and eating habits. The Uygur often eat more carnivorous, love oil Nang, pilaf, tea and other greasy food, the Uygur men often drink (liquor based), smoking is also more common, these are likely to affect the incidence. Secondly, because the GerdQ symptom questionnaire itself factors, different patients have different understanding and expression of heartburn, acid reflux symptoms. As well as the impact of local language and education, which may have a certain impact on the results of the GerdQ score.

The proportion of patients with RE group rise up with the increasing of GerdQ score from 0 to 14, which Bai's [7] research had the same results. So patients with more severe reflux symptoms are more likely to have RE. Further study showed that the risk of RE will be relatively small when the GerdQ total score $<8$, and none of the patients with a GerdQ score of 0-2 had RE. This finding is in agreement with previous study [5]. It shows that the lower the GerdQ score, the smaller of the risk of suffering from RE. A higher score signifies a greater possibility of RE.

This study shows that GerdQ average score is higher in the RE group than that in the non-RE group, the score of esophageal erosion was significantly higher than that of the normal controls, GerdQ score has a good value to distinguish the RE group and the non-RE group. The higher the score, the greater possibility of suffering from RE.

For the RE patients, the majority of them (90.71\%) were suffering from grade A or grade B, and this result is consistent with the results of $\mathrm{Ma}$ et al [17], which found $96.9 \%$ of Chinese patients with RE had LA grade A or grade B lesion. This suggests RE patients tend to be less severe in Chinese patients than it is in Western population. The study also showed that the GerdQ score increased with increasing grade of RE. Spearman rank correlation analysis showed that the GerdQ score was positively correlated with the severity of $\mathrm{RE}(\mathrm{P}<0.01)$. Further study showed that there was a direct correlation between the GerdQ score and the severity of RE in the Uygur and Han. That is, the higher the score, the more severe esophageal mucosal erosion. The result is same to the Uygur and Han. It indicated that the symptom score of $\mathrm{RE}$ has clinical diagnostic value, we can rely on the GerdQ score to distinguish the RE grading. It is consistent with the results of Zhai et al [18]. But Li et al [19] and Pace et al [20] found that there was no correlation between GerdQ score and severity of RE. The reasons for the difference may be related to the ethnic and geographical differences of the subjects in the study. This study still has a guiding significance for the diagnosis of RE in Chinese population.

We found that the optimal GerdQ cutoff score for RE in our study was 9, corresponding to a sensitivity of $87.7 \%$ and a specificity of $65.7 \%$ for the diagnosis of RE, which is the same as the study of Jonasson et al [8], who reported a sensitivity of $66 \%$ and a specificity of $64 \%$ if the cut-off value of GerdQ score is increased to 9. However, in a previous studied by Jones et al [5] indicated that the sensitivity and specificity were $65 \%$ and $71 \%$, respectively, the diagnosis of GERD with a GerdQ cut-off value of $\geq 8$, and other studies have shown a sensitivity for GerdQ of 57$78 \%$ and specificity of $46-50 \%$ for the diagnosis of GERD when the GerdQ score was $\geq 8[21,22,8]$. The difference in the sensitivity and specificity of GerdQ for the diagnosis of RE may be attributed to the following reasons: In this study, all the patients enrolled were Uygur and Han in Xinjiang region, the difference of the way of life, economic status, geographical environment and other factors.

The present study has several strengths: (1) There were many studies about the application of GerdQ score in the diagnosis of gastroesophageal reflux disease, but no independent study about the correlation between GerdQ score and the severity of RE, our study will be useful in the diagnosis of RE, for which current data is largely lacking; (2) This study analyzed the correlation between GerdQ score and the severity of RE for Uygur and Han come to the same conclusion, which made the conclusion more convincing. Therefore, our findings are of great significance in guiding clinical practice.

\section{MATERIALS AND METHODS}

\section{Study population}

Data from consecutive Uygur and Han patients who were suspected of RE in the medical centre of the People's Hospital of Xinjiang Uygur Autonomous Region were prospectively collected from August 1, 2014 to December 31, 2015. Inclusion criteria: (1) Patients with heartburn and (or) regurgitation as the main symptoms manifested over the last 4 weeks; (2) Patients with symptoms suggestive of RE, who underwent first diagnostic; (3) Adult (age $\geq 18$ years) male or female; (4) Educational level above elementary school, able to fill out the questionnaire independently; (5) Written informed consent. Exclusion criteria: (1) Patients with alarm symptoms such as weight loss, dysphagia, gastrointestinal bleeding, anemia, hematemesis, melena, etc; (2) Take any proton pump inhibitors, histamine 2 receptor antagonists, or gastrointestinal motility drugs for more than 2 weeks before inclusion; (3) Underwent upper gastrointestinal bleeding, a history of gastric surgery, esophageal stenosis, peptic ulcer, gastric, esophageal varices, or malignant tumors in 1 year; (4) Gastroscopy contraindications or refusing endoscopy ; (5) Patients with severe cardiovascular, pulmonary, liver and kidney diseases, or other severe mental diseases; (6) Pregnancy and lactating women; (7) History of taking non steroidal anti-inflammatory drugs; (8) History of alcohol or drug abuse; and (9) patients were diagnosed with $\mathrm{RE}$ and during treatment.

\section{Study design}

Research steps: All of the Eligible patients provided written informed consent followed by completion of the 
GerdQ, blinded to the investigator, and a GerdQ sum score was calculated. Endoscopy was then performed. GerdQ was completed blinded to the endoscopic doctor. The patient who had esophagocardiac mucosal erosions were detected by endoscopy would be classified according to the Los Angeles classification system. The study protocol was approved by the Ethics Review Committee of Xinjiang Uygur Autonomous Region of China.

Questionnaire: The questionnaires, which comprised the General data questionnaire and the Chinese version of GerdQ, demographic information (gender, age, ethnic, height, weight, educational level and so on). The GerdQ is a symptom scale (Table 3), which including heartburn, regurgitation, epigastric pain, nausea, sleep disturbance, and use of over the counter (OTC) drugs. Patients were asked to review the frequencies of various symptoms during last week, and which were described by a Likert scale from 0 to 3 for positive symptom problems and from 3 to 0 for negative symptom problems, with a total GerdQ score range from 0 to18.

Endoscopy: All patients were required to fast for at least $12 \mathrm{~h}$ prior to the conventional endoscopy (Olympus-260, Tokyo, Japan). The severity of RE was graded according to the Los Angeles classification system (LA grading) [11]. Grade A: one or more mucosal break less than $5 \mathrm{~mm}$ long, which doesn't extend between the tops of two mucosal folds. Grade B:one or more mucosal break more than 5mm long, which doesn't extend between the tops of two mucosal folds. Grade C: one or more mucosal break that is continuous between the tops of two or more mucosal folds but which involves less than $75 \%$ of the oesophageal circumference. Grade D: one or more mucosal break which involves more than $75 \%$ of the oesophageal circumference.

The diagnostic standard of RE: Take endoscopy as the gold standard for the diagnosis of RE. All participants were divided into patients with RE and those with non-RE according to whether they had or had not esophagocardiac mucosal break or erosions found by endoscopy.

\section{Statistical analysis}

Statistical analyses were performed using SPSS software (version 17.0). All data are expressed as mean \pm standard deviation (SD), percentages and ranges. The measurement data and numeration data were assessed by t-test and chi-square test respectively. and Spearman rank correlation analysis was used for ranked data. ROC curve was used to determine the optimal cut-off for the diagnosis of RE. In all analyses, $P<0.05$ was considered statistically significant.

\section{CONCLUSIONS}

The GerdQ score has good value to distinguish RE patients from non-RE patients. GerdQ score was positively correlated with the severity of RE. The higher of the score, the more severe of the esophageal mucosal erosion.
Further analysis for the Uygur and Han we both come to the same conclusion. Although RE cannot be accurately diagnosed by the GerdQ score alone in patients suspected of RE, it can be regarded as a useful tool for screening RE in the general population. A definitive diagnosis of RE still depends on endoscopy.

\section{Author contributions}

The study was designed by FG, MW and JZZ. The manuscript was first written by MW, JZZ and then revised by XJK. XLH, AK, YXL and XLH collected the materials. MW and JZZ have contributed equally to this work. All authors reviewed and approved the final manuscript.

\section{ETHICS APPROVAL AND CONSENT TO PARTICIPATE}

Not applicable.

\section{AVAILABILITY OF DATA AND MATERIAL}

Data sharing not applicable to this article as no datasets were generated or analysed during the current study.

\section{CONFLICTS OF INTEREST}

We state that we have no conflicts to disclose.

\section{FUNDING}

This work supported by the National Natural Science Foundation of China (81160052). No roles of the funding body are in the design of the study and collection, analysis, and interpretation of data and in writing the manuscript.

\section{REFERENCES}

1. He J, Ma X, Zhao Y, Wang R, Yan X, Yan H, Yin P, Kang X, Fang J, Hao Y, Li Q, Dent J, Sung JJ, et al. A population-based survey of the epidemiology of symptomsdefined gastroesophageal reflux disease: the systematic investigation of gastrointestinal disease in China. BMC Gastroenterol. 2010; 10:94.

2. El-Serag HB, Sweet S, Winchester CC, Dent J. Update on the epidemiology of gastro-oesophageal reflux disease: a systematic review. Gut. 2014; 63:871-880.

3. Henry MA. Diagnosis and management of gastroesophageal reflux disease. Arq Bras Cir Dig. 2014; 27:210-215.

4. Dent J, Jones R, Vakil N, Halling K, Junghard O, Wernersson B. A management strategy for GERD base on 
the gastroesephageal reflux disease questionnaire (GerdQ). Scand J Gastroenterol. 2008; 43:34-35.

5. Jones $\mathrm{R}$, Junghard $\mathrm{O}$, Dent $\mathrm{J}$, Vakil $\mathrm{N}$, Halling $\mathrm{K}$, Wernersson B, Lind T. Development of the GerdQ, a tool for the diagnosis and management of gastro-oesophageal reflux disease in primary care. Aliment Pharmacol Ther. 2009; 30:1030-1038.

6. Jonasson C, Moum B, Bang C, Andersen KR, Hatlebakk JG. Randomised clinical trial: a comparison between a GerdQ-based algorithm and an endoscopy-based approach for the diagnosis and initial treatment of GERD. Aliment Pharmacol Ther. 2012; 35:1290-1300.

7. Bai Y, Du Y, Zou D, Jin Z, Zhan X, Li ZS, Yang Y, Liu Y, Zhang S, Qian J, Zhou L, Hao J, Chen D, et al. Gastroesophageal Reflux Disease Questionnaire (GerdQ) in real-world practice. A national multicenter survey on 8065 patients. J Gastroenterol Hepatol. 2013; 28:626-631.

8. Jonasson C, Wernersson B, Hoff DA, Hatlebakk JG. Validation of the GerdQ questionnaire for the diagnosis of gastrooesophageal reflux disease. Aliment Pharmacol Ther. 2013; 37:564-572.

9. Suzuki H, Matsuzaki J, Okada S, Hirata K, Fukuhara S, Hibi T. Validation of the GerdQ questionnaire for the management of gastrooesophageal reflux disease in Japan. United European Gastroenterol J. 2013; 1:175-183.

10. Zavala-Gonzales MA, Azamar-Jacome AA, MeixueiroDaza A, Ramos A, J JR, Roesch-Dietlen F, RemesTroche JM. Validation and diagnostic usefulness of gastroesophageal reflux disease questionnaire in a primary Care Level in Mexico. J Neurogastroenterol Motil. 2014; 20:475-482.

11. Lundell LR, Dent J, Bennett JR, Blum AL, Armstrong D, Galmiche JP, Johnson F, Hongo M, Richter JE, Spechler SJ, Tytgat GN, Wallin L. Endoscopic assessment of oesophagitis: clinical and functional correlates and further validation of the Los Angeles classification. Gut. 1999; 45:172-180.

12. Tack J, Becher A, Mulligan C, Johnson DA. Systematic review: the burden of disruptive gastro-oesophageal reflux disease on health-related quality of life. Aliment Pharmacol Ther. 2012; 35:1257-1266.
13. Peery AF, Dellon ES, Lund J, Crockett SD, McGowan CE, Bulsiewicz WJ, Gangarosa LM, Thiny MT, Stizenberg K, Morgan DR, Ringel Y, Kim HP, Dibonaventura MD, et al. Burden of gastrointestinal disease in the United States: 2012 update. Gastroenterology. 2012; 143:1179-1187.

14. Vakil N, van Zanten SV, Kahrilas P, Dent J, Jones R; Global Consensus Group. The Montreal definition and classification of gastroesophageal reflux disease: a global evidence-based consensus. Am J Gastroenterol. 2006; 101:1900-1920.

15. Hu ZY, Zhou LY, Lin SR, Xue Y. Analysis of clinical characteristics in 2088 patients with reflux esophagitis during past decade. Chin J Dig. 2005; 25:717-719.

16. Munila A, Niu CY, Minawaer HJ. An investigation for gastroesophageal reflux disease of Han Chinese and Uygur in Urumqi. J Xinjiang Med Univ. 2012; 35:1257-1261.

17. Ma XQ, Cao Y, Wang R, Yan X, Zhao Y, Zou D. Prevalence of, and factors associated with, gastroesophageal reflux disease: a population-based study in Shanghai, China. Dis Esophagus. 2009; 22:317-322.

18. Zhai HL, Wen Y. GerdQ in the diagnosis of gastroesophageal reflux disease. J Chin Physician. 2013; 15:697-699.

19. Li Y, Li CF, Yan ZK, Wang LQ, Zhang XF, Zhang B. The value of gastroesophageal reflux disease questionnaire in the diagnosis of gastroesophageal reflux disease. Chin J Dig Endosc. 2014; 31:336-338.

20. Pace F, Bianchi Porro G. Gstreesophageal reflux disease: a typieal spectrum disease (a new conceptual framework is not needed). Am J Gastroenterol. 2004; 99:946-949.

21. Zhou LY, Wang Y, Lu JJ, Lin L, Cui RL, Zhang HJ, Xue Y, Ding SG, Lin SR. Accuracy of diagnosing gastroesophageal reflux disease by GerdQ, esophageal impedancemonitoring and histology. J Dig Dis. 2014; 15:230-238.

22. Lacy BE, Chehade R, Crowell MD. A prospective study to compare a symptom-based reflux disease questionnaire to 48 - $\mathrm{h}$ wireless $\mathrm{pH}$ monitoring for the identification of gastroesophageal reflux (revised 2-26-11). Am J Gastroenterol. 2011; 106:1604-1611. 